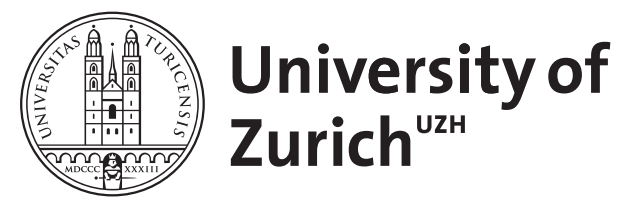

\title{
Sicherheit. Anspruch-Versprechen-Utopie
}

\author{
Magyar-Haas, V ; Grube, N
}

\begin{abstract}
Im Rahmen des Artikels wird das Phänomen der Unsicherheit auf Basis der Plessnerschen anthropologischen Perspektive aufgezeigt und der Umgang mit diesem Phänomen fokussiert: expliziert werden der unsicherheitskompensierende, utopische Gehalt von Selbst- und Gemeinschaftsentwürfen sowie gesellschaftspolitische Techniken. Ausgehend von den Schein-Handlungssicherheiten erzeugenden Maßnahmen im Kontext der Sozialen Arbeit wird das Sicherheitsversprechen in dem gesellschaftlichen Zusammenhang zwischen Markt, Staat und Zukunftsprognosen machttheoretisch verortet. Weiterhin wird aufgezeigt, wie diese paradoxalen Verflechtungen das anthropologische Bedürfnis, Verlangen nach bzw. Anspruch auf Sicherheit, das Versprechen dieser Sicherheit mittels politischer Maßnahmen sowie die faktische Nichteinlösbarkeit dieses Versprechens, die Utopie der Sicherheit, eine Sicherheitsspirale generieren.
\end{abstract}

DOI: https://doi.org/10.1007/s12592-009-0012-4

Posted at the Zurich Open Repository and Archive, University of Zurich

ZORA URL: https://doi.org/10.5167/uzh-27805

Journal Article

Published Version

Originally published at:

Magyar-Haas, V; Grube, N (2009). Sicherheit. Anspruch-Versprechen-Utopie. Soziale Passagen. Journal für Empirie und Theorie sozialer Arbeit, 1(1):35-48.

DOI: https://doi.org/10.1007/s12592-009-0012-4 


\title{
Sicherheit. Anspruch - Versprechen - Utopie
}

\author{
Veronika Magyar-Haas • Norbert Grube
}

Zusammenfassung: Im Rahmen des Artikels wird das Phänomen der Unsicherheit auf Basis der Plessnerschen anthropologischen Perspektive aufgezeigt und der Umgang mit diesem Phänomen fokussiert: expliziert werden der unsicherheitskompensierende, utopische Gehalt von Selbst- und Gemeinschaftsentwürfen sowie gesellschaftspolitische Techniken. Ausgehend von den Schein-Handlungssicherheiten erzeugenden Maßnahmen im Kontext der Sozialen Arbeit wird das Sicherheitsversprechen in dem gesellschaftlichen Zusammenhang zwischen Markt, Staat und Zukunftsprognosen machttheoretisch verortet. Weiterhin wird aufgezeigt, wie diese paradoxalen Verflechtungen: das anthropologische Bedürfnis, Verlangen nach bzw. Anspruch auf Sicherheit, das Versprechen dieser Sicherheit mittels politischer Maßnahmen sowie die faktische Nichteinlösbarkeit dieses Versprechens, die Utopie der Sicherheit, eine Sicherheitsspirale generieren.

Schlüsselwörter: Anthropologie $\cdot$ Machttheorie $\cdot$ Soziale Arbeit $\cdot$ Professionalität $\cdot$ Sicherheit Utopie

\section{Certainty. Entitlement - assurance - utopia}

\begin{abstract}
Referring to Helmuth Plessner's anthropological perspective, this article focuses on the phenomenon of uncertainty and analyzes the handling of this phenomenon. The utopian content of concepts of the self and the community, its meaning as a compensation for missing certainty, and socio-political techniques to offset the perceived lack of certainty will be elaborated. Referring to pseudo-confidence generating measurements in the context of social work the promise of certainty will be embedded in a perspective of the theory of power within the social relation between market, government and prognoses. Furthermore it will be shown how paradoxical interrelations, i.e. the anthropological need, requirement and desire for certainty on the one hand and the governmental promise of certainty as well as the non-feasibility of this promise, like a utopia of certainty, on the other hand, create a spiral of certainty.
\end{abstract}

Keywords: Anthropology · Theory of power - Social work · Professionalism · Certainty · Utopia

(C) VS-Verlag 2009

V. Magyar-Haas M.A. $(\bowtie)$

Pädagogisches Institut, Lehrstuhl Allgemeine Pädagogik, Universität Zürich, Freiestrasse 36,

8032 Zürich, Schweiz

E-Mail: vmagyar@paed.uzh.ch

Dr. N. Grube

Institut für Historische Bildungsforschung (IHBF) Pestalozzianum, Pädagogische Hochschule Zürich,

Kurvenstrasse 17, 8090 Zürich, Schweiz

E-Mail: norbert.grube@phzh.ch 


\section{Unsicherheit als anthropologische Konstante ${ }^{1}$}

Helmuth Plessner bestimmt in seinen Schriften zur politischen Anthropologie ${ }^{2}$ den Menschen als von Natur aus offenes, unergründliches Wesen und legt die Unbestimmtheitsrelation als grundlegende Voraussetzung des geschichtlichen und politischen menschlichen Daseins (Arlt 1996, S. 17) fest. Die paradoxe Lage des Menschen, die Handlungsunsicherheit mit der gleichzeitigen Sicherheitssuche und sein utopischer Standort, ergibt sich nach dem Anthropologen aus dem wesenstypischen Ausdruck der exzentrischen Positionalität des Menschen, welche er der zentrischen Positionalität der Tiere gegenüber abgrenzt. Plessner bestimmt in den „Stufen des Organischen und der Mensch“ den Menschen als Lebewesen, welches gleichzeitig in und außerhalb einer Umwelt positioniert ist, in einer zentrischen und exzentrischen Position. Mittels der Exzentrizität ist er in der Lage, ,sich von sich zu distanzieren, zwischen sich und seine Erlebnisse eine Kluft zu setzen. Dann ist es diesseits und jenseits der Kluft, gebunden im Körper, gebunden in der Seele und zugleich nirgends, ortlos außer aller Bindung von Raum und Zeit und so ist er Mensch“ (Plessner [1928] 2003, S. 290). Als exzentrisch positioniertes, von Natur aus gebrochenes Wesen, das nicht in sich ruht, sondern immerzu außerhalb seiner selbst steht, zeigen sich menschliche Selbstbezüge immerzu von einem Moment des Entzugs und der Verfehlung gekennzeichnet, welches gleichzeitig die Verflechtung des Selbst mit Anderem begründet. Die Nicht-Koinzidenz des Subjekts mit sich, sein Leben im Modus der Indirektheit und partiellen Selbstverfehltheit sind es, die als maßgeblicher Beweggrund intersubjektiver Vollzüge fungieren. Aus dieser existentiellen Gebrochenheit des Menschen ergeben sich nach Plessner die Fragen, wie: „Was soll ich tun, wie soll ich leben“, welche die gesamte Philosophiegeschichte ebenfalls durchziehen und welchen keine „,noch so naive, naturnahe, ungebrochene, daseinsfrohe und traditionsgebundene Epoche der Menschheit sich entwinden konnte. Wohl hat es Zeiten gegeben (und wird auch wieder Zeiten geben), die nicht davon sprachen und in denen das Bewusstsein der konstitutiven Heimatlosigkeit des menschlichen Wesens durch starke Bindungen an Scholle und Familie, an Haus und Ahnen überdeckt war. [...] Die Idee des Paradieses, des Standes der Unschuld, des goldenen Zeitalters, ohne die noch keine menschliche Generation gelebt hat (heute heißt die Idee: Gemeinschaft) ist der Beweis für das, was dem Menschen fehlt, und für das Wissen darum [...].“ (Plessner [1928] 2003, S. 383)

Plessner baut seinen Ansatz auf die These auf, dass jedes Ding , aufgrund“ oder ,kraft eines ontologischen Doppelaspektes, aufgrund einer Relation zwischen materieller Substanz, welche als Quasi-,Innerlichkeit', als ,Kern'vorausgesetzt wird, und den unmittelbar wahrnehmbaren Eigenschaften erscheint. Dieses ,Innere‘, die Seele, beschreibt Plessner

1 Dieser Beitrag ist die überarbeitete Fassung eines an der Theorie AG in Bielefeld am 5.12.2008 zur Diskussion gestellten Vortrags. Der Aufsatz beansprucht nicht, abgerundete Forschungsergebnisse zu präsentieren, vielmehr das Phänomen der Sicherheit aus unterschiedlichen theoretischen Perspektiven kritisch zu hinterfragen und dadurch eine weiterführende Debatte einzuleiten.

2 vgl. dazu „Grenzen der Gemeinschaft. Eine Kritik des sozialen Radikalismus“ (1924) sowie „Macht und menschliche Natur. Ein Versuch zur Anthropologie der geschichtlichen Weltansicht" (1931). 
in seiner Zweideutigkeit: „Ihre Geheimnisse weichen vor jedem Versuch der Enträtselung in andere Tiefen zurück. Jedes Seelische hat also eine Bestimmtheit, die Laune, der Schmerz, die Liebe, das echte Gefühl, die falsche Freude lassen sich fassen, aber erfasst zerrinnen sie unter dem Griff der Wahrnehmung, wie wir erwachen, wenn wir träumen, dass wir träumen“ (Plessner [1924] 2003, S. 62). Dieses Innere, welches Sein und Werden zugleich ist, wehrt sich einerseits gegen jegliche Festlegung, es bedarf aber auch des authentischen Gesehenwerdens. Den Drang nach Offenbarung, die Geltungsbedürftigkeit sowie den Drang nach Verhaltung, die Schamhaftigkeit, beschreibt Plessner als Resultate der ontologischen Zweideutigkeit. „Wir wollen uns sehen und gesehen werden, wie wir sind, und wir wollen ebenso uns verhüllen und unbekannt bleiben, denn hinter jeder Bestimmtheit unseres Seins schlummern die unsagbaren Möglichkeiten des Andersseins“ (Plessner [1924] 2003, S. 63), wie Plessner in der Schrift „Grenzen der Gemeinschaft“ expliziert. Um die konstitutive Heimatlosigkeit, Wurzellosigkeit, die „unerträgliche Exzentrizität seines Wesens“, die „Hälftenhaftigkeit“ der eigenen Lebensform, woraus die Handlungsunsicherheit notgedrungen resultiert, zu kompensieren, sei der Mensch auf Kultur und künstliche Formen des Handelns angewiesen (Plessner [1928] 2003, S. 385). Die konstitutive Gleichgewichtslosigkeit seiner besonderen Positionalitätsart und nicht erst die Störung eines ursprünglich normal, harmonisch gewesenen und wieder harmonisch werden könnenden Lebenssystems sei der ,Anlass ${ }^{6}$ zur Kultur sowie zur Künstlichkeit. Auch wenn Plessner den Menschen als offenes Wesen konstruiert, worin die Stärke und pädagogische Anschlussfähigkeit seines anthropologischen Ansatzes begründet liegt, betont er die Notwendigkeit der situativen Festlegung, des Handlungsvollzugs in jeder offenen, sozialen Situation, wonach die Unsicherheit in situ gehandhabt werden muss, ohne ein letztendliches, festgelegtes Sicherheitskonzept zu bedienen.

\section{Unsicherheit als Professionalitätskriterium}

Diese anthropologisch zurückführbare grundsätzliche Ungewissheit und Uneindeutigkeit menschlichen Denkens und Handelns ist in ihren Konsequenzen für die soziale Arbeit zu reflektieren und zu analysieren. So hat aus einer historisch-geschlechtertheoretischen Perspektive und ausgehend von der Krise traditioneller Frauenberufe Ursula Rabe-Kleberg (1996, S. 276 ff.) die Frage nach der Professionalität von beruflichem Handeln und beruflichen Strukturen neu gestellt und das Handeln unter Bedingungen der Unsicherheit als Spezifikum des (sozial)pädagogischen Handlungsfeldes aufgezeigt. Die in diesem Kontext als Dienst erbrachten Leistungen zeichnen sich dadurch aus, dass sie primär nicht materiell seien, größtenteils aus Interaktionsprozessen bestünden und daher unstetig und wenig standardisierbar seien. Dass Ungewissheit und Unsicherheit als Strukturmerkmal dieser Form der Dienstleistungsarbeit fungieren, resultiert aus dem Verhältnis von Funktion und Ziel beziehungsweise von Mittel und Ziel dieser Tätigkeit. Einerseits sei die Dienstleistung nicht von der „Unwägbarkeit des personalen Faktors“ (Rabe-Kleberg 1996, S. 294) zu lösen, wodurch die Mittel der Dienstleistung nicht mit technischen Regeln bestimmbar seien. Andererseits liege die Funktion der weiblich konnotierten Erziehungs- und Pflegearbeit primär in der Vermeidung, in der Abwehr der Risiken und Störungen, woraus sich keine expliziten Zielvorstellungen ableiten ließen, da diese höchstens nur ex negativo 
definiert werden könnten. Die Zielformulierungen bleiben demnach undefiniert, unbestimmt und werden in den Arbeitsprozess verlagert. Um mit struktureller Ungewissheit und Unsicherheit dennoch verantwortungsvoll umzugehen, fordert Rabe-Kleberg (1996, S. 295) Qualifikationen, Generierung neuer Kompetenzen sowie professionelle Bedingungen ein. Einen Beitrag zur Lösung des Ungewissheitsdilemmas liefert Abbott (1988) im Rahmen seines Modells professionellen Arbeitens, indem er dieses im Spannungsdreieck Diagnose - Schlussfolgerung - Anwendung verortet. „Diagnosis and treatment are mediating acts: diagnosis takes information into the professional knowledge system and treatment brings instructions back out from it. Inference, by contrast, is a purely professional act. It takes the information of diagnosis and indicates a range of treatments with their predicted outcomes“. (Abbott 1988, S. 40). Auf diesem reflexiven Handlungskonzept basierend wäre Ungewissheit in jedem Handlungsvollzug temporär überwunden. Dieses idealtypische Modell nutzt das Wissen sowie die Einschätzbarkeit von Konsequenzen als Legitimation der professionell und verantwortungsvoll Handelnden und berücksichtigt weder die gesellschaftlichen Normen, noch die soziale und institutionelle Kontrolle, welche einerseits aus dem Eingebundensein professionellen Handelns in Organisationen und andererseits aus dem gesellschaftlichen Auftrag des institutionalisierten „Helfens“ resultiert (Rabe-Kleberg 1996, S. 296). Auf Basis dieses Eingebundenseins der Dienstleistung in ökonomisch-bürokratischen Strukturen entsteht nach Claus Offe (1984, S. 15 f.) ein Spannungsverhältnis zwischen den unterschiedlichen Rationalitäten: die ökonomischbürokratische Rationalität erfordere Handlungseffektivität und Effizienzkontrolle, welche der oben dargestellten Dienstleistungsrationalität zuwiderläuft. Dass die Ungewissheitsproblematik ein konstituierendes Merkmal der Struktur sozialpädagogischer, professioneller Arbeit sei, lässt sich weiterhin mit dem aus dem sogenannten „doppelten Mandat““ resultierenden Spannungsverhältnis zwischen gesellschaftlichen Normvorstellungen und individuellen, fallspezifischen Besonderheiten, zwischen Standardisierung und Differenzierung begründen. Professionalität wäre demnach die Fähigkeit und die Bereitschaft, die widersprüchlichen Strukturen professioneller Arbeit „nicht nur zu ertragen, sondern aktiv zu balancieren“" (Rabe-Kleberg 1996, S. 298).

Mittels der Konzepte wie „Evidence Based Practice“ werden Sicherheitsversprechen formuliert und eingeführt, welche dazu beitragen sollen, die genannte, für professionelles Handeln konstitutive Balancierung von Unsicherheiten zu reduzieren. Hierzu zählen weiterhin das wissenschaftlich generierte Wissen, welches als Begründung und Legitimation professionellen Handelns fungiert und nicht im Sinne eines Transfers oder einer Transformation in professionelles Handlungswissen überführt werden kann (Dewe et al. 1992), ebenso wie die divergenten Präventionsmaßnahmen, etwa „soziale“ Frühwarnsysteme zur frühzeitigen Erkennung riskanter Entwicklungen und Lebenslagen ${ }^{3}$. Das Attribut „,soziale“ scheint hier unerlässlich, erinnere sonst der Terminus „Frühwarnsystem“ allein schnell an den militärischen Kontext, an Aufklärungsflugzeuge, Militärsatelliten und Luftraumüberwachung. Nach einer Pilotphase von 2001-2004 implementierte das Familienministerium des Landes Nordrhein-Westfalen im Rahmen des Projektes „Frühe Hilfen für Kinder und Familien“" bis 2006 an 34 Standorten ein soziales Frühwarnsystem, Tendenz steigend.

3 vgl. dazu sowie zu den weiteren Ausführungen das Projekt „Frühes Erkennen von riskanten Entwicklungen“" unter http://www.soziale-fruehwarnsysteme.de/ 
Der politische Unsicherheitsbearbeitungsdrang scheint erheblich und führt zu entsprechenden Sicherheitstechniken. Neben einer Vielzahl anderer Frühwarnsysteme ${ }^{4}$ sei vor allem auf den medizinischen Bereich verwiesen (Sarasin 2003), auf die Krebsvorsorgeuntersuchungen, die diskutierten verpflichtenden Kinderfrühuntersuchungen U1 bis U10, mit Konsequenzen für die Eltern bei Nichteinhaltung der Fürsorgepflicht dieser Art. Somit bleibt zwar der Körper im sicherheitspolitischen Fokus, doch wird später zu zeigen sein, dass es verkürzt ist, das gouvernementale Sicherheitsdispositiv als einverleibt oder inkorporiert zu betrachten und den an der gesamten Bevölkerung ausgerichteten und aufzeigbaren Steuerungsanspruch außer Acht zu lassen.

Cindi Katz widmet sich in ihrem Artikel „Der Terror des Wachsamkeitswahns. Sicherheit und Kinderschutzindustrie in den Vorstädten“ (20045) den von der Kinderschutzindustrie angebotenen Überwachungstechnologien für das Eigenheim, wie in Teddybären oder Luftreinigungsgeräten versteckbare Nanny Cams, Child Watch Monitors und Home Security Systeme, die von einer „Kultur der Angst“ (Katz 2004) zehren. Katz zeigt auf, wie dieses gut funktionierende, bereits 2004 1,1 Milliarden US\$ starke und stets wachsende Geschäft der Eigenheimüberwachungsindustrie eine „Farce“ erzeugt beziehungsweise die Absicht verschleiert, „das Eigenheim durch sinnlose Selbstverteidigungstechnologien, die es gegen unterschiedliche Formen des Eindringens wappnen sollen, zu einer Zitadelle zu machen“ (Katz 2004). Die angebotenen Überwachungstechnologien sollen den Eltern wie den Kindern ein Gefühl von Kontrolle und Sicherheit geben. Das Verhältnis von Bedürfnis und Bedarfsbefriedigung oder eher Angebot und Nachfrage wird in diesem Sicherheitskontext besonders verwoben, denke man an die mehreren hunderttausendfach verkauften Exemplare von Büchern wie „Lob der Disziplin“ oder „Warum unsere Kinder Tyrannen werden“. Diese außerordentlichen Verkaufszahlen für angebliche Fachbücher lassen durchaus auf ein Sicherheitsbedürfnis von Eltern, Erziehern und Lehrern schließen. Publikationen, welche sich einer Rhetorik des Verfalls kultureller Normen und Werte bedienen, liefern einfachste, klare, entkontextualisierte, scheinbar allgemeingültige Patentrezepte für den guten und richtigen Umgang mit den „kleinen Despoten“ (Winterhoff) und erzeugen dadurch eine Art Pseudo-Sicherheit bezüglich des erzieherischen, normadäquaten und normaufrechterhaltenden Handelns (Langenohl et al. 2007, S. 189-207).

\section{Gouvernementale Sicherheitsversprechen als Machttechnik}

Diese Verfallsrhetorik kultureller Werte und Normen lässt sich als Anschluss an gouvernementale Machtstrategien mit ausgeprägten gesellschaftlichen Lenkungs- und Regulierungsambitionen betrachten, welche der dargelegten Auffassung des Menschen

4 Frühwarnsysteme finden sich gerade auch mit paramilitärischer Konnotation im Bereich des Naturkatastrophenschutzes (Tsunami, Erdbeben, Hurrican, Unwetter, etc.) und in der Außenpolitik, in der auswärtige Kulturpolitik und humanitäre Aktionen als Mittel gelten, um religiös, ethnisch oder wirtschaftlich motivierte Konflikte rasch zu erkennen oder sogar zu vermeiden, dazu vgl. Hoffmann, 1996.

5 http://www.widersprueche-zeitschrift.de/article1264.html 
mit dem Charakteristikum der ontologischen Ort- und Gleichgewichtslosigkeit diametral entgegenstehen. Gouvernementale Sicherheitsversprechen, hier verstanden als Teil eines Sicherheitsdispositivs, das als Instrument zur Bevölkerungssteuerung zu verstehen ist (Foucault 2001, S. 285-289; Foucault 2004, S. 38), bilden den Kontext für die skizzierten Sicherheitsparadoxien in der sozialen Arbeit, die wiederum als Teil gouvernementaler Strategien angesehen werden kann. Die Kombination der bislang anthropologisch fokussierten Untersuchung mit einer machttheoretisch fundierten Perspektive bietet daher eine ergänzende Vertiefung der Analyse von Sicherheitsansprüchen und versprechungen und vermag sowohl sicherheitspolitische Ambitionen und Techniken, als auch die in der ontologischen Zweideutigkeit begründeten Grenzen der Bevölkerungslenkung bzw. die „Grenzen der Ordnung“, so die Titelthese einer Plessner und Foucault zusammenführenden Untersuchung (Richter 2005), analytisch in den Blick nehmen. Das Sicherheitsdispositiv des 19. und 20. Jahrhunderts richtet sich im Unterschied zur in begrenzten Räumen erfolgten Disziplinierung des Körpers, etwa beim Arbeiten, Essen, Waschen oder sogar Schlafen in den frühneuzeitlichen Zucht- und Waisenhäusern, primär an die gesamte Gesellschaft, an die gesamte Bevölkerung zumeist innerhalb eines Nationalstaats. Die Machttechnik des gouvernementalen Sicherheitsversprechens zielte im wesentlichen auf die Vermeidung von Risiken durch Kalkulation und statistische Berechnung von Gefahren, wie Naturkatastrophen (Wetteraufzeichnungen) oder Krankheiten (Foucault 2001, S. 290 f.). Sicherheit stand im Konnex mit Effizienz und dem Regulativ des Marktes, um Wettbewerb, Mobilisierung von Arbeits- und Wirtschaftskraft, Optimierung von Lebensleistung zu gewährleisten sowie ,Zirkulation zuzulassen (...) von Leuten (...) und Waren“ (Foucault 2004, S. 52). Entsprechend galt es nach Foucault vor allem im 19. und 20. Jahrhundert, mit präventiv angelegten Sicherheitsrahmungen den größtmöglichen Teil der Bevölkerung zugunsten des Marktes zu präparieren und einzusetzen, galt doch der Markt mit zunehmender gouvernementaler Selbstbeschränkung als Ort des gesellschaftlichen Regulativs.

\section{Sicherheitsrhetorik}

Die Ausrichtung der Bevölkerung auf den Markt wird gegenwärtig vor allem Schulen zugewiesen (Tyack u. Cuban 2001, S. 39; Meyer u. Ramirez 2005, S. 217, 219) und mitunter auch der sozialen Arbeit. Von ihnen verspricht man sich die Qualifikation zur effizienten, eigenverantwortlichen Teilhabe in Beruf und Wirtschaft ebenso, wie die Vermehrung der Akzeptanz des Marktes als gesellschaftliches Regulativ. Ähnliche Ziele verfolgen auch um Vertrauen und damit um unreflektierte Zustimmung werbende politische Sicherheitsversprechen zur Stabilisierung des Systems der sozialen Marktwirtschaft, die häufig - etwa auf Wahlkampfplakaten - mit Ankündigungen der Wohlstandswahrung oder -mehrung zugunsten einer besseren, gleichwohl in den vorgegebenen Bahnen eingehegten Zukunftsgestaltung kombiniert waren. Generell dienen Sicherheitsversprechen bis heute als zentrales Mittel zum politischen Machterhalt und -erwerb (Mergel 2007, S. 377). Vor allem die bürgerlichen Parteien CDU/CSU und FDP proklamierten sie in Wahlkampfslogans, wie „Keine Experimente“ (1957), „Unsere Sicherheit. CDU“ (1965), 
„Weiter so“ (CDU, 1987) oder jüngst bei der niedersächsischen Landtagswahl „Sicher leben, geborgen fühlen. Sicherheit! Besser CDU“ (2003). ${ }^{6}$

\section{Beobachtung, Prognose, Intervention, Prävention als Machttechniken}

Sicherheitsversprechen verblieben jedoch nicht nur auf der Ebene politischer Rhetorik, sondern sie dienten zur Legitimation bevölkerungspolitischer Interventionen. Versteht man unter Öffentlichkeit mit Plessner den „Inbegriff von Möglichkeitsbeziehungen“ und „eine sozialformende Macht ersten Ranges“"(Plessner [1924] 2003, S. 51), so beanspruchten Regierungen, wissenschaftliche Berater, Unternehmer sowohl in den auf pseudo-demokratische Akklamation beruhenden Diktaturen, als auch in den parlamentarischen Demokratien des 20. Jahrhunderts, diese soziale Formung zu leiten. Denn „das Interesse an möglichster Minderung sozialen Risikos ist infolgedessen eine beständige Kraftquelle sozialer Gestaltung“(Plessner [1924] 2003, S. 51). Machthaber verschiedener Regime versuchten diese Minderung sozialen Risikos durch die Nutzung von Beobachtung, Prognose, Intervention zum Ziele der Prävention zu lenken. Diese Abfolge erinnert an Abbotts modellhaftes Spannungsdreieck der Sozialen Arbeit und steht im Zentrum der folgenden Abschnitte. Soziale Formung erfolgte allerdings im 20. Jahrhundert mit unterschiedlichsten Mitteln, etwa der Selektion, Exklusivität und Integration, und stieß in Teilen durchaus auf entsprechende Erwartungen gesellschaftlicher Gruppen. Eine frühe sozialpsychologische Studie über die Attraktivität des Nationalsozialismus betonte vor allem die Sicherheit und die soziale Fürsorge, die verbreitete Ordnungsbedürfnisse der Bevölkerung beruhigten (Neumann u. Noelle 1954, S. 21). Das Sicherheitsversprechen, eingebettet in der volksgemeinschaftlichen Pseudo-Geborgenheit, sollte den Verlust politischer Rechte aufwiegen und rassistische Leistungsauslese und Menschenzucht vernebeln (Frei 2002, S. 106, 162). Doch auch die Selektion, die Aussonderung vermeintlich Asozialer stieß selbst in der Erinnerung auf partielle Zustimmung, etwa die Polizeiaktion im Frühjahr 1933 gegen die beunruhigend als „Gemeinschaftsfremde“ (Peukert 1982) wahrgenommenen Bettler.

Sicherheitsversprechen und -aktionen beruhten also auch historisch betrachtet häufig auf entsprechend gelagerte Erwartungen in der Bevölkerung, etwa nach Preis- und Arbeitsplatzstabilität und Vermeidung neuer vernichtender Kriege fast durchgängig seit den 1950er Jahren. Sie sollten somit und auch durch das Generieren von Unsicherheit das Machtsystem stabilisieren. Nach Plessner ([1924] 2003, S. 30) bedeutet

6 Politische Kontinuitäts- und Sicherheitsversprechen sind so dominant, dass Willy Brandts Regierungserklärungsankündigung „Mehr Demokratie wagen“ von 1969 heute gleichsam zum Gegenentwurf stilisiert wird (Brandt 1969), der jedoch nur sehr gemäßigt nachgeahmt wurde von Gerhard Schröders Bekundung 1998, ,nicht alles anders, sondern vieles besser machen zu wollen“. Andeutungen eines zu heftigen Wandels, gar alternativer Programme werden weitgehend vermieden - mit Ausnahme der Grünen in den 1980er Jahren. Das in amerikanischen Präsidentschaftswahlkämpfen nahezu obligatorische „Change“ als Schlachtruf des Herausforderers ist in Deutschland in dieser harten begrifflichen Alleinstellung nahezu undenkbar. Selbst die „Wende“ Helmut Kohls 1982/83 wurde erst dann zunehmend verbalisiert, als der Machtwechsel bereits im Herbst 1982 vollzogen war. 
„die wirtschaftliche Gesichertheit Zufriedenheit mit den Sicherungsfaktoren, d.h. mit den bestehenden Zuständen“. Die an den Erwartungen der Bevölkerung ausgerichtete wie zugleich appellierende gouvernementale Sicherheitspolitik wurde häufig ergänzt um emotionalisierende Appelle an Angst und Sorgen. Sicherheit benötigte ein Feindbild (Juden, Ausländer, Homosexuelle, Bettler oder auch des Antikommunismus), um die eigene „Ordnung“ nicht zu hinterfragen beziehungsweise bestätigen zu können. Dies wurde paradoxerweise erst generiert durch partielle Inszenierung von Angst (Bauman 2008, S. 67-68; Sarasin 2003). Als etwa der Antikommunismus in den ausgehenden 1950er Jahren langsam aufzuweichen drohte, bemühten sich die gouvernementalen Kommunikationsstrategen um immer neue, das Feindbild verhärtende Sicherheitsversprechen (Grube 2005, S. 304 f.). Dieser Kreislauf hielt bis in die 1980er Jahre an und kreierte eine Art Pseudo-Sicherheit, die jedoch gerade deshalb nur scheinbar Ängste milderte, weil die Versprechen Ängste instrumentalisierten, somit aber den Zwang zu immer mehr Sicherheit und damit weitergehende Sicherheitsversprechen beförderten. Eine gerade den Kalten Krieg kennzeichnende Sicherheitsspirale oder gouvernemental geprägte Sicherheitsutopie zeichnet sich ab.

\section{Kontrolle der Möglichkeitsbeziehungen durch Bevölkerungsbeobachtungen}

Im Gegensatz zur sozialistischen Utopie völliger Gleichheit, versuchte man im Westen Zukunftsunsicherheiten durch eine Politik der kleinen Sicherheitsschritte einzuhegen, die auf intensiver Bevölkerungsbeobachtung beruhte. Sie umfasste Messungen konjunktureller Verläufe und des Verbraucherverhaltens zur Marktoptimierung ebenso, wie die Beobachtung und Therapie abweichenden sozialen Verhaltens. So galt es, Sicherheitsansprüche zu beobachten durch quantitative Bevölkerungsbefragungen und sozialstatistische Datenreihen, um Wissen zu generieren über Ängste, Hoffnungen/Befürchtungen, Sorgen, Unzufriedenheiten. Nichts wird so sehr vermessen wie die „Ängste der Deutschen“ - so der Titel einer jüngsten Studie der Gesellschaft für Konsumforschung - Ängste vor Preissteigerungen, Naturkatastrophen, Krankheiten, Ausländerzuzug, Kriminalität und Terror, zuletzt ermittelt auch im Datenreport 2008 des Statistischen Bundesamtes und des Wissenschaftszentrums Berlin (WZB) (Datenreport 2008, S. 417). Unmengen von Datenbanken und Informationsnetzen, deren Erkenntnispotentiale und Datenmengen zumeist unausgeschöpft bleiben, sorgen für eine bevölkerungspolitisch nutzbare „Dauerbeobachtung“, so lautet die Kennzeichnung des Internetlinks, der auf Daten des seit 1978 erhobenen Wohlfahrtssurveys verweist.

Gerade in dieser sozialstatistischen Vermessung wird deutlich, wie sehr sich das Sicherheitsversprechen an die Bevölkerung als Ganzes wendet, welche im Zentrum einer nationalstaatlichen Verwaltung von sozialer Sicherheit mit immer schärferer Bedarfsprüfung und intensiven Datenerfassungen bleibt.

Die Dauerbeobachtung von Ängsten und immer neue Sicherheitsversprechen bedeuten nun nicht, dass jahrhundertalte, generationenübergreifend tradierte Internalisierungen ordnungsgemäßen, affektreduzierten Verhaltens keine Geltung erlangt hätten. Verschiedene Sicherheit gewährende Mechanismen sind in der westlichen Sphäre weitgehend 
einverleibt (Elias 1990, S. 318-328, 333). ${ }^{7}$ Zugleich erhöht die Bindung an Gesetze oder zeremonielle Normen und Formen die „Sicherheit und Würde des Benehmens“ (Plessner [1924] 2003, S. 78). Doch Internalisierungen, eingeübte Verhaltensweisen unterliegen dem Druck von Dynamiken der arbeitsteiligen Gesellschaft und bedürfen in diesen vielfältigen Verflechtungszusammenhängen der permanenten Überprüfung, Variation und Adaption. Dieser „Balanceakt“ der „Beziehung von Individuum und sozialer Welt“ (Berger u. Luckmann 2004, S. 145, 154) mit seinen zahlreichen offenen Ausprägungen ist aus gouvernementaler Sicht zu beobachten und zu dirigieren.

\section{Prognosen als Vorraussetzung für bevölkerungspolitische Interventionen}

Die westliche Politik bedurfte der Beobachtung, um die „Seelen der Massen“ (Arendt 1996, S. 744-5, 763) auf das jeweils dominierende Gesellschafts- und Wirtschaftssystem hin zu lenken. Damit wurden vagabundierende Sorgen und Ängste aufgegriffen, um sie zu dämpfen, wenn sie volkswirtschaftlicher Entfaltung entgegen standen, aber auch um sie eventuell zu schüren, etwa im Bereich der inneren und äußeren Sicherheit. Diesen paradox anmutenden Sicherheitsversprechen nachzukommen, bedurfte es der auf Bevölkerungsvermessungen beruhenden Erstellung von Prognosen. Sie sollten aufzeigen, in welche Richtung der Möglichkeitsbeziehungen Teile der Gesellschaft tendierten, um Zukunftsoptionen kalkulierbar einzuhegen, „Gleichgewichtszustände und Regelmäßigkeiten“ (Foucault 2001, S. 291) herzustellen und Zugriff zu erlauben auf Projektionen, Sehnsüchte und Wünsche der Bevölkerung, die - wenn die sozialpsychologische Weitung der Plessnerschen Anthropologie erlaubt ist - die ,goldene Zukunft oder Wiederkehr goldener Vergangenheit“" ersehne (Plessner [1924] 2003, S. 30). Ähnlich wie bei Prognosen im Rahmen der sozialen Arbeit sollen sozialempirisch grundierte, bevölkerungsbezogene Prognosen Wissen vermitteln über Einstellungen und Verhaltensweisen sowie über die Notwendigkeit zu ihrer Transformation. So diente z. B. das 1964 vom Allensbacher Institut für Demoskopie erstellte „Gesellschaftsbild 1970“ dazu, den ermittelten Ängsten vor einem Atomkrieg, unheilbaren Krankheiten und Unberechenbarkeiten des sozialen Schicksals mit der Betonung der sozialen Aufstiegsmöglichkeiten und der Generierung eines neuen Sozialbewusstseins zugunsten der bestehenden Marktbedingungen zu begegnen. Dies sei eine politisch-psychologische Führungsaufgabe der Bundesregierung zur Widerlegung der ,Zukunftsphantastik“ in modernen Diktaturen (Allensbach 1964, S. 145, 155-6). Sie bezieht sich allerdings eher auf eine von der Gegenwart bestimmte zukünftige Machbarkeit und gleicht damit der von Bauman skizzierten Aufgabe des Jägers, die gegenwärtige Wildbahn im Gleichgewicht zu halten - im Gegensatz zum Typ des die Natur, die Welt gestaltenden Gärtners, der nach Bauman bis weit ins 20. Jahrhundert hinein für Utopien stand (Bauman 2001, S. 140-142).

7 Hier ist etwa auf die verbreitete Akzeptanz des staatlichen Gewaltmonopols oder Regeln im Straßenverkehr zu verweisen, ebenso auf eingeübte Rituale und Zeremonielle, die unkontrollierte, Mitmenschen schädigende Trieb gesteuerte Handlungen und Gewalttaten dämpfen, allerdings auf Kosten der Ausbildung einer Selbstzwangapparatur. 
Politische Machthaber waren und sind zunehmend selbst verunsichert (Riesman 1961, S. 138, 225), ob und wie sie die als unübersichtlich wahrgenommene soziale Vielgestalt Öffentlichkeit dirigieren konnten. Sie benötigten Prognosen, um entsprechende bevölkerungspolitische Interventionen zu erlassen. Sie waren nicht nur auf die Ausschöpfung des Humankapitals unter dem Primat der Effizienz und des Marktes (Foucault 2004) ausgerichtet. Vielmehr zielten sie auf die Befestigung von auf Vertrauen basierender gesellschaftlicher Geschlossenheit, sahen diese gerade konservative Politiker doch besonders in der zweiten Hälfte des 20. Jahrhunderts im Systemkonflikt des Kalten Krieges im stets beobachteten sozialistischen Kollektiv realisiert und betrachteten die Multioptionen der westlichen Gesellschaft eher als Gefährdung denn als Chance (Neumann 1966; Kleinmann 1995, S. 155-6, 280, 328). ${ }^{8}$ Auch jüngst werden Sicherheitsversprechen geprägt von Appellen an Geschlossenheit, etwa bei Parteien zur Erhöhung der Wahlchancen (Bergmann 2002, S. 98), oder an Vertrauen als Bindemittel zwischen Wählern und Regierungen, Aktionären und Aktienmarkt, Konsumenten und Produzenten. Sie weisen auf ganzheitliche, eher gemeinschaftliche Ordnungsvorstellungen hin, etwa im Sinne Plessners ([1924] 2003, S. 45): „Immer ist Gemeinschaft kreishaft gegen ein unbestimmtes Milieu abgeschlossene Sphäre der Vertrautheit. Ihr wesensnotwendiger Gegenspieler, ..., von dem sie sich abhebt, ist die Öffentlichkeit, der Inbegriff von Leuten und Dingen, die nicht mehr „dazugehören“, mit denen aber gerechnet werden muss.“ Vertrauen einwerbende, gar einklagende Gefolgschafts- oder Gemeinschaftsappelle tendieren zu Mimesis und Konformität, wie Marcuse (1967, S. 30) kritisierte. Skepsis vor der Vertrauensrhetorik in politischen und wirtschaftlichen Krisen scheint angebracht, betrachtet man alarmistische Warnungen vor Vertrauenserosionen gegenüber politischen Institutionen oder Finanzmärkten. Dass das Mißtrauen und damit auch der temporäre Konflikt eine konstruktive Kategorie politisch denkender und handelnder autonomer Menschen sein könnte, kommt in diesen Sicherheitsdiskursen nicht vor (Skinner 2006).

\section{Interventions- und Präventionszuweisungen an Schulen und Sozialarbeit}

Um sowohl soziale Geschlossenheit und Vertrauen wie auch Markteffizienz herzustellen, wird vor allem auf die Schule, auf Bildungs- und Familienpolitik als langfristig wirkende Interventionsinstanzen verwiesen (Meyer u. Ramirez 2005, S. 219) und ihnen im Gegensatz zur kurzfristig wirkenden Steuer- und Arbeitsmarktpolitik Präventionsfunktion zugesprochen. Schule und auch jüngste Vermessungen von Schulleistungen sollen helfen, in der Weltwettbewerbsgesellschaft Zukunft zu gestalten und damit die Sicherheit der Gesellschaft vor wirtschaftlichen Abstürzen oder Kriminalität zu gewährleisten. Scheitern diese hohen Ambitionen, wird in westlichen Staaten ein erhöhtes Sicherheitsrisiko ausgerufen, mit den plakativen Mahnungen der „Youth at risk“ oder gar der „Nation at Risk“, so der Titel des unter der Präsidentschaft von Ronald Reagan verfassten „Reports on American Education“ 1983 (Tröhler 2006). Dieser Alarm über neue Gefährdungen

8 Vertrauen im Sinne von Gefolgschaft ist allerdings nicht mehr in Foucaults Sicherheitsdispositiv zu verorten, sondern tendiert eher zu Carl Schmitts Politikprämissen (Richter 2005, S. 25; Frevert 2003, S. 53 f.). 
nationaler Wettbewerbsfähigkeit durch vermeintlich leistungsschwache Jugendliche lässt sich sowohl in den 1950er Jahren anläßlich der Debatte über die sogenannten ,Halbstarken' verfolgen, wie dann vor allem in den schon angesprochenen Wertewandeldebatten der 1970er Jahre. Auch heute folgt diesem Alarm stets der Ruf nach noch intensiverer Schulbildung, nach noch mehr Prävention und, frühzeitige Betreuung von , Problemfällen durch Sozialarbeiter an den Schulen (...), um die Chancen gering qualifizierter Jugendlicher zu erhöhen“. So lautete der Tenor der Allensbacher Umfrage „Mehr Job-Chancen für Jugendliche. Was muss die Politik tun?“" unter Arbeitgebern, Sozialarbeitern und Haupt- und Förderschullehrern vom Juni 2008 im Auftrag der neoliberalen, vom Arbeitgeberverband Gesamtmetall finanzierten und Wirtschaftslobbyisten geführten „Initiative Neue Soziale Marktwirtschaft“. In dieser Studie werden vorrangig die „Motivations- und Persönlichkeitsdefizite der gering qualifizierten Jugendlichen“ für deren Ausschluss aus dem Ausbildungsmarkt verantwortlich gemacht und nicht ,ausbildungsunwillige Unternehmer" (INSM 2008, Pressemitteilung; vgl. Gammelin u. Hamann 2005). Sozialarbeit wird hier als Sicherheitstechnologie in den Kontext bevölkerungspolitischer Sicherheitsversprechen gestellt.

\section{Die Sicherheitsspirale}

Der Zwang nach immer intensiverer Beobachtung , gefährdeter Jugendlicher` und die hektische Abfolge immer neuer Studien und Forderungen nach Interventionen und Präventionen beschleunigen eine Spirale von immer neuen Sicherheitsversprechen und steigenden Erwartungshaltungen, mit immer neuen Interventionsmaßnahmen und ebenso neue Enttäuschungen (Bauman 2008, S. 21). Diese Sicherheitsspirale verheißt zwar Fortschritte, bewegt sich aber gleichsam seitwärts in einem Wellental von Befürchtungen, Versprechungen und Pseudo-Sicherheiten. Das gouvernemental geprägte Sicherheitsdispositiv als bevölkerungspolitische Intervention zur Optimierung von menschlicher Leistungskraft ist keineswegs so effektiv, wie Foucaults Deutung neoliberaler Politik andeutet. Denn Deterritorialisierungsprozesse, wie weltweite Kommunikation, wirtschaftliche Transaktionen und militärische Interventionen, sprengen das bislang vorwiegend nationalstaatlich orientierte Sicherheitsdispositiv. Gouvernementale Sicherheitsrhetoriken bleiben zwar bestehen. Doch modifizierten sie sich mit der Beschränkung des Sozialstaates in den letzten Jahren in Richtung einer neuen Eigenverantwortungsrhetorik. In deren Gefolge, wie anhand der Eigenheimüberwachungsindustrie gezeigt, ,,weitet sich der private Markt für Sicherheitsbelange mächtig aus“ (Rose 2000, S. 98). Zugleich verfestigen sich jedoch gerade in wirtschaftlichen Krisenzeiten staatliche Sicherheitsversprechen. Sie werden um so utopischer, je mehr die eigentlich Planbarkeit und Prävention verheißenden Diagnosen und Prognosen, etwa über Konjunkturverläufe oder demographische Entwicklungen, in die Irre führen. Denn die Möglichkeitsbeziehungen der Gesellschaft, die Ambivalenzen und Paradoxien der menschlichen Seele zwischen dem Drang nach Entäußerung und nach Verhüllung (Plessner, [1924] 2003, S. 63), dem Wunsch, ,sich anderen Menschen zu nähern als auch sich von ihnen abzuschließen“ (Riesman 1961, S. 87) können nicht valide erfaßt werden. Beobachtung als bevölkerungspolitische Machttechnik kann kaum die permanente, vielschichtige, zukunftsoffene und rasch wechselnde Verhältnishaftigkeit 
oder Reziprozität von außen geleiteten Charakteren in der von ihnen bestimmten Öffentlichkeit (Riesman 1961) erfassen. Somit können auch nicht die gesellschaftlichen Handlungsspielräume in ihren Möglichkeiten und Grenzen hinreichend ermessen werden.

Die ontologische Zweideutigkeit und die Unzulänglichkeit der Diagnose- bzw. Messinstrumente lassen die Sicherheitstechniken in Frage stellen. Da diese wesenstypische Hälftenhaftigkeit dennoch kompensiert werden muss, entstehen die Sicherheitsbedürfnisse immer drängender. Carolyn Taylor und Sue White kritisieren in ihrem Artikel „Knowledge and Reasoning in Social Work: Educating for Humane Judgement“ (2006) die Konzentration und Fixierung auf das rein formale Wissen, welche außer Acht ließe, dass diese Art von Wissenssicherheit die Praktiker zu vorschnellen Urteilen verleite in Situationen, wo eine ,respectful uncertainty“ sich als angemessener erweisen könnte. Bezogen auf die Soziale Arbeit könnte diese ,respectful uncertainty“ als Ausweg aus der Sicherheitsspirale, welche durch neue Präventionstechnologien und mechanisch anmutende Handlungssicherheitsversprechen stets weiter hochgetrieben wird, angesehen werden. Aus diesem Grund wäre es ertragreich, anstatt der Generierung von Sicherheitsversprechen auf ideologischer Basis auf der empirischen Ebene, mittels weiterführender, vertiefender ethnographischer Studien und Analysen, zu erschließen, wie Professionelle im Kontext der Sozialen Arbeit mit Handlungsunsicherheiten umgehen, wie sie die durch Unsicherheit gekennzeichneten Situationen bewältigen.

\section{Literatur}

Abbott, A. (1988). The systems of profession. An essay on the division of expert labour. Chicago, London: University of Chicago Press.

Arendt, H. (1996). Elemente und Ursprünge totaler Herrschaft. Antisemitismus, Imperialismus, totale Herrschaft. 5. Auflage der Taschenbuchausgabe. München: Piper.

Arlt, G. (1996). Anthropologie und Politik. Ein Schlüssel zum Werk Helmuth Plessners. München: Fink.

Baumann, Z. (2008). Flüchtige Zeiten. Leben in der Ungewissheit. Hamburg: Hamburger Edition.

Berger, P. L. \& Luckmann, Th. (2004). Die gesellschaftliche Konstruktion der Wirklichkeit: eine Theorie der Wissenssoziologie. Frankfurt am Main: Fischer-TB.

Bergmann, K. (2002). Der Bundestagswahlkampf 1998. Vorgeschichte, Strategien, Ergebnis. Wiesbaden: Westdeutscher Verlag.

Brandt, W. (1969/1979). Regierungserklärung des Bundeskanzlers Willy Brandt vom 28. Oktober 1969. In Die großen Regierungserklärungen der deutschen Bundeskanzler von Adenauer bis Schmidt. Eingeleitet und kommentiert von Klaus von Beyme (S. 251-281). München/Wien.

Datenreport 2008. Ein Sozialbericht für die Bundesrepublik Deutschland, hg. v. Statistischen Bundesamt/GESIS/ZUMA/WZB. Bonn: Bundeszentrale für politische Bildung.

Dewe, B., Ferchhoff, W. \& Radke, F.-O. (1992). Das „Professionswissen“ von Pädagogen. Ein wissenstheoretischer Rekonstruktionsversuch. In B. Dewe et al. (Hrsg.), Erziehen als Profession. Zur Logik professionellen Handelns in pädagogischen Feldern (S. 70-91). Opladen: Leske + Budrich.

Elias, N. (1990). Über den Prozess der Zivilisation. Soziogenetische und psychogenetische Untersuchungen. Bd. 2 Wandlungen der Gesellschaft. Entwurf zu einer Theorie der Zivilisation. Frankfurt/Main: Suhrkamp, 15. Auflage.

Foucault, M. (2001). In Verteidigung der Gesellschaft. Vorlesungen am Collège de France (197576). Frankfurt/Main: Suhrkamp. 
Foucault, M. (2004). Geschichte der Gouvernementalität Bd. 1: Sicherheit, Territorium, Bevölkerung. Frankfurt/Main: Suhrkamp.

Frei, N. (2002). Der Führerstaat. Nationalsozialistische Herrschaft 1933 bis 1945. München: DTV, 7. Auflage.

Frevert, U. (2003). Vertrauen - eine historische Spurensuche. In Dies. (Hrsg.), Vertrauen. Historische Annäherungen (S. 7-66), Göttingen: Vandenhoeck \& Ruprecht.

Gammelin, C. \& Hamann, C. (2005). Die Strippenzieher. Manager, Minister, Medien-wie Deutschland regiert wird. Berlin: Econ.

Grube, N. (2005). Die Darstellung Deutschlands durch die semi-staatliche Auslandsnachrichtenagentur „Deutsche Korrespondenz“ 1951-1967. In J. Paulmann (Hrsg.), Auswärtige Repräsentationen. Deutsche Kulturdiplomatie nach 1945 (S. 289-311). Köln: Böhlau.

Hoffmann, H. (1996). „Dritte Säule“ der Außenpolitik. Zur aktuellen Diskussion um die auswärtige Kulturpolitik. Internationale Politik, 3, 15-20.

Initiative Neue Soziale Marktwirtschaft (2008). Pressekonferenz. Mehr Job-Chancen für Jugendliche: Was muss die Politik tun? / Pressemitteilung. http://www.insm.de/Umfragen__ Studien. html.

Institut für Demoskopie Allensbach (1964). Das Gesellschaftsbild 1970. Über die Entstehung eines neuen Sozialbewußtseins in der Bundesrepublik.

Katz, C. (2004). Der Terror des Wachsamkeitswahns. Sicherheit und Kinderschutzindustrie in den Vorstädten. Widersprüche, (92): http://www.widersprueche-zeitschrift.de/article1264.html

Kleinmann, H.-O. (Bearb.) (1995). Heinrich Krone. Tagebücher. Bd. 1: 1945-1961. Düsseldorf: Droste.

Langenohl, S., Lauer, N., Magyar-Haas, V., Oelkers, N., Tamuleviciute, A. \& Thieme, H. (2007). Wider die undemokratischen Tendenzen der neuen Disziplinierung. Neue Praxis, 2, 189-207.

Marcuse, H. (1967). Der eindimensionale Mensch. Studien zur Ideologie der fortgeschrittenen Industriegesellschaft. Neuwied/Berlin: Luchterhand.

Mergel, Th. (2007). Verkaufen wie Zahnpasta? Politisches Marketing in den bundesdeutschen Wahlkämpfen, 1949-1990. In H. Berghoff (Hrsg.), Marketinggeschichte. Die Genese einer modernen Sozialtechnik (S. 372-399). Frankfurt/Main.

Meyer, J. E. \& Ramirez, F. (2005). Die globale Institutionalisierung der Bildung. In J. W. Meyer, G. Krücken (Hrsg.), Weltkultur. Wie die westlichen Prinzipien die Welt durchdringen (S. 212234). Frankfurt/Main: Suhrkamp.

Neumann, E. P. \& Noelle, E. (1954). Antworten. Politik im Kraftfeld der öffentlichen Meinung. Allensbach: Verlag für Demoskopie.

Neumann, E. P. (1966). Der gute Wolf. Über den Verzicht auf Propaganda. Die politische Meinung 11 (114), 3-5.

Noelle-Neumann, E. (1978). Werden wir alle Proletarier? Wertewandel in unserer Gesellschaft. Zürich, Osnabrück: Edition interfrom.

Noelle-Neumann, E. \& Köcher, R. (1988). Die verletzte Nation. Über den Versuch der Deutschen, ihren Charakter zu ändern. Stuttgart: DVA, 2. Auflage.

Offe, Cl. (1984). Arbeit als soziologische Schlüsselindustrie? In Cl. Offe (Hrsg.), „Arbeitsgesellschaft". Strukturprobleme und Zukunftsperspektiven (S. 13-43). Frankfurt/Main, New York: Campus.

Peukert, D. (1982). Volksgenossen und Gemeinschaftsfremde. Anpassung, Ausmerze und Aufbegehren unter dem Nationalsozialismus. Köln.

Plessner, H. (1924). Grenzen der Gemeinschaft. Eine Kritik des sozialen Radikalismus. Bonn: Verlag Friedrich Cohen.

Plessner, H. (1928). Die Stufen des Organischen und der Mensch. Einleitung in die philosophische Anthropologie. In Dux et al. (Hrsg.), Helmuth Plessner. Gesammelte Schriften IV. 
Plessner, H. (1931). Macht und menschliche Natur. Ein Versuch zur Anthropologie der geschichtlichen Weltansicht. In Dux et al. (Hrsg.), Helmuth Plessner. Gesammelte Schriften V (S. 135234) Bielefeld.

Rabe-Kleberg, U. (1996). Professionalität und Geschlechterverhältnis. Oder: Was ist „semi“ an traditionellen Frauenberufen? In C. Arno \& W. Helsper (Hrsg.), Pädagogische Professionalität. Untersuchungen zum Typus pädagogischen Handelns (S. 267-392). Frankfurt/Main: Suhrkamp.

Riesman, D. (1961). Die einsame Masse. Eine Untersuchung der Wandlungen des amerikanischen Charakters. Hamburg: Rowohlt.

Richter, N. A. (2005). Grenzen der Ordnung. Bausteine einer Philosophie des politischen Handelns nach Plessner und Foucault. Frankfurt/Main: Campus.

Rose, N. (2000). Tod des Sozialen? Eine Neubestimmung der Grenzen des Regierens. In U. Bröckling, S. Krasmann \& Th. Lemke (Hrsg.), Gouvernementalität der Gegenwart. Studien zur Ökonomisierung des Sozialen (S. 72-109). Frankfurt/Main.

Sarasin, Ph. (2003). Zweierlei Rassismus? Die Selektion des Fremden als Problem in Michel Foucaults Verbindung von Biopolitik und Rassismus. In M. Stingelin (Hrsg.), Biopolitik und Rassismus (nach Foucault). Was leben soll und was sterben muß (S. 55-79). Frankfurt/Main: Suhrkamp.

Skinner, Q. (2006). Sind wir modernen Bürger wirklich alle Sklaven? Interview mit Jürgen Kaube. Frankfurter Allgemeine Zeitung, 15.12.2006. S. 48.

Taylor, C. \& White, S. (2006). Knowledge and reasoning in social work: Educating for humane judgement. The British Journal of Social Work, 36 (6), 927-954.

Tröhler, D. (2006). Öffentliche Schule, Governance und Demokratie. In G. Miller-Kipp \& B. Zymek (Hrsg.), Politik in der Bildungsgeschichte - Befunde, Prozesse, Diskurse (S. 87-100). Bad Heilbrunn: Verlag Julius Klinkhardt.

Tyack, D. \& Cuban, L. (2001). Tinkering toward Utopia. A century of public school reform. Cambridge, Mass.: Harvard University Press. 